Medicine, which Zhang co-founded, and that is, obviously, not a participant in this agreement. (Doudna was also a co-founder, but is no longer involved with the company.) Even so, with the intent of extending the technology's impact as widely as possible, the Broad Institute has recently thrown its weight behind an initiative to create a worldwide CRISPR-Cas9 licensing pool. The initiative, organized by MPEG LA, a packager of patent pools, would act as a one-stop shop for organizations seeking to incorporate patented CRISPR-based technology into their products. The Broad, along with Harvard University and the Massachusetts Institute of Technology, both of Cambridge, and The Rockefeller University, of New York, have jointly submitted ten patent families for consideration to the Denver-based MPEG LA, which originally cut its teeth in developing a patent pool model for consumer electronics.

The Broad's patent sharing proposal focuses on non-therapeutic applications of genome editing, as these areas are better suited to the non-exclusive terms inherent in patent pool licensing. "The field of therapeutics, human therapeutics particularly, would be more difficult to address with a pool," says Kristin Neuman, executive director of biotech licensing at MPEG LA. Diagnostics, industrial biotech and agricultural biotech are all areas in which the concept could work. "There will be a large pool of patents. Licensees will probably be able to license core sets of those patents for a given application," she says.

Of the companies contacted by Nature Biotechnology, ERS Genomics declined to comment on its intentions; Caribou Biosciences is non-committal. "Caribou is evaluating a variety of different options for making the CRISPR-Cas9 technology broadly available for the research community and for developers of products across multiple different markets," a spokesperson for the company says. Even without buy-in from all companies, Neuman says MPEG LA's could still take off. Those working on MPEG LA are gathering as many patents for the pool as possible, and Newman is in contact with "all of the main patent holders," she says. It will probably take at least a year before the scheme is open for licensing.

The patent landscape surrounding CRISPR-based genome editing technology is already becoming crowded, and will continue to get more so, as other scientists and biotech firms join the rush to build a position in this still emerging market. Parisbased Cellectis recently secured a European patent (EP3004337) for using RNA-guided endonucleases, such as Cas9 or Cpf1, to genetically engineer $\mathrm{T}$ cells. "It encompasses every type of chimeric nuclease," says Cellectis CEO and chairman André Choulika.

This patent covering T-cell editing could have significant implications for CAR-T cell therapy developers, given a recent finding that a CD19-directed chimeric antigen receptor (CAR)-T cell therapy, developed using CRISPR-Cas9, showed greater potency in a mouse model of acute lymphoblastic leukemia than the same therapy engineered through conventional recombinant methods (Nature 543, 113-117, 2017). The lead author on that study, Justin Eyquem, now at Memorial Sloan Kettering Cancer Center, in New York, was previously a researcher at Cellectis, although he is not named among the inventors on the patent disclosure. Although the innovation may seem obvious in 2017, says Choulika, "it was not obvious to anyone" when Cellectis began work in this area several years ago. The patent has a filing date of April 1, 2014, and a priority date of May 29, 2013.

It was a 'dead' version of Cas9 that secured patent rights for the life sciences arm of Merck KGA. The Darmstadt, Germanybased pharma's wholly owned subsidiary MilliporeSigma, received both European and Australian patents for its 'proxy-CRISPR' version of the genome editing system. The technique employs a Cas9 endonuclease derived from Francisella novicida, FnCas9, along with a catalytically inactivated form of the conventional Streptococcus pyogenes Cas9 (SpCas9). This 'dead' Cas9 binds to a site proximal to the FnCas 9 target site, and is thought to enhance the genome editing efficiency of the system by altering local chromatin structures and enabling FnCas9 to function (Nat. Comm. 8, 14958, 2017). The approach, Merck claims, opens up regions of the genome previously inaccessible to CRISPR-based editing and is applicable to a range of different CRISPRbased systems and endonucleases, including FnCpf1. "The most common reagent in our proxy-CRISPR toolkits will likely be deadSpCas9, serving as a generalized reagent to boost the activity of any other CRISPR system with which a researcher might have trouble," says a Merck spokesperson.

For now, CRISPR Therapeutics, Intellia Therapeutics and Editas Medicine are the leaders in developing therapeutic applications of CRISPR. As the technology evolves, however, their IP positions might be eroded by new editing techniques that fall outside existing patent claims. "I'm not sure that the entire technology has been discovered," Fuller says.

Cormac Sheridan Dublin

\section{Massachusetts injects more cash into jobs}

Massachusetts will provide up to $\$ 500$ million over five years to support the state's booming biotech industry, including investments in public infrastructure, R\&D, workforce training and education. The funding will include up to $\$ 295$ million in capital funding aimed at infrastructure improvements and up to $\$ 150$ million in tax incentives to encourage job creation, according to a June 17 press release from the state administration. Two days after the announcement, Governor Charlie Baker discussed the plan at the $2017 \mathrm{BIO}$ International Convention in San Diego. It is "the next act in our investment in the life sciences," he said. A big part of the investment will be around workforce development. "That is going to end up being a major place where I believe (we) will create public-private partnerships going forward," he said. The new commitment comes ten years after thengovernor Deval Patrick used the BIO meeting to unveil his vision for a ten-year, \$1-billion Supercluster program to expand life sciences activities in the state (Nat. Biotechnol. 25, 604, 2007). Legislation passed a year later established the Massachusetts Life Sciences Center (MLSC) to implement the program. MLSC, which will continue to administer the new funding, has invested more than $\$ 650$ million across the state, including through infrastructure grants and tax incentives and capital spending to spur job growth. According to Ernst \& Young's latest annual Beyond Borders industry analysis, New England biotechs (those with revenues less than $\$ 500$ million) raised $\$ 7.1$ billion in capital in 2016 , compared with $\$ 4.8$ billion for biotechs in the San Francisco area and $\$ 2.5$ billion for those in Far East Asia.

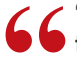

"I will be Novartis' biggest cheerleader today." Kite's CEO Arie Belldegrun writes of his excitement at the US FDA's advisory committee decision to give the green light to tisagenlecleucel, an anti-CD19 chimeric antigen receptor (CAR)-T cell therapy, and its significance as a milestone in the technology's maturation. (Kite World Blog, 12 July 2017)

"I am highly skeptical that gene editing in the human germline can make sense as a safe and more effective approach than embryo screening by PGD [prenatal genetic diagnosis] and PGS [preimplantation genetic screening]." UC Davis stem cell researcher Paul Knoepfler weighs in on the editing of a heart disease gene in human embryos, published in Nature on August 2. (The Niche, 2 August 2017)

"We're now at a moment in history where we don't have to follow Darwin's rules. We need to be conscious of that." A philosophical take from Juan Carlos Izpisua Belmonte of the Salk Institute in La Jolla, California, who created pig-human chimeras, and is part of the team that reported in August on editing human embryos. (STAT News, 7 August 2017) 